Geach Christopher (Orcid ID: 0000-0002-6335-7483)

Fritts David, C. (Orcid ID: 0000-0002-6402-105X)

Kaifler Bernd (Orcid ID: 0000-0002-5891-242X)

Kaifler Natalie (Orcid ID: 0000-0002-3118-6480)

Kjellstrand Carl, Bjorn (Orcid ID: 0000-0003-3777-3886)

Williams Bifford, P. (Orcid ID: 0000-0002-1797-5985)

Eckermann Stephen, D. (Orcid ID: 0000-0002-8534-1909)

\title{
Gravity Wave Breaking and Vortex Ring Formation Observed by PMC Turbo
}

C. Geach ${ }^{1}$, S. Hanany ${ }^{1}$, D. C. Fritts ${ }^{2}$, B. Kaifler ${ }^{3}$, N. Kaifler ${ }^{3}$, C. B. Kjellstrand ${ }^{4}$, B. P. Williams $^{2}$, S.D. Eckermann ${ }^{5}$, A. D. Miller ${ }^{6} \dagger$, G. Jones ${ }^{7} \dagger$, J. Reimuller ${ }^{8}$

${ }^{1}$ University of Minnesota, Twin-Cities, Minneapolis, MN, USA

${ }^{2}$ GATS, Boulder, CO, USA

${ }^{3}$ German Aerospace Center (DLR), Munich, Germany

${ }^{4}$ Columbia University, New York, NY, USA

${ }^{5}$ Space Science Division, U.S. Naval Research Laboratory, Washington, DC, USA

${ }^{6}$ University of Southern California, Los Angeles, CA, USA

${ }^{7}$ Rigetti Computing, Berkeley, CA, USA

${ }^{8}$ Integrated Spaceflight Services, Boulder, CO, USA

Corresponding author: Christopher Geach (geach003@umn.edu)

$\dagger$ Formerly at Columbia University, New York, NY, USA

Index terms: 3332, 3334, 3379, 3384

Key Words: Convective instability, Gravity wave breaking, Vortex rings, Polar mesospheric clouds, Mesosphere

\section{Key Points:}

- Gravity wave breaking and associated vortex rings are observed in imaging and lidar data of polar mesospheric clouds

- Analysis of the data gives parameters of the gravity wave and rates of energy and momentum transfer due to its breaking

- Observed vortex ring parameters agree with predictions by numerical resolutions of gravity wave breaking

This article has been accepted for publication and undergone full peer review but has not been through the copyediting, typesetting, pagination and proofreading process which may lead to differences between this version and the Version of Record. Please cite this article as doi: 10.1029/2020JD033038 


\begin{abstract}
Polar mesospheric cloud (PMC) imaging and lidar profiling performed aboard the 5.9 day PMC Turbo balloon flight from Sweden to northern Canada in July 2018 revealed a wide variety of gravity wave $(\mathrm{GW})$ and instability events occurring nearly continuously at approximately $82 \mathrm{~km}$. We describe one event exhibiting GW breaking and associated vortex rings driven by apparent convective instability. Using PMC Turbo imaging with spatial and temporal resolution of $20 \mathrm{~m}$ and $2 \mathrm{~s}$, respectively, we quantify the GW horizontal wavelength, propagation direction, and apparent phase speed. We identify vortex rings with diameters of 2$5 \mathrm{~km}$ and horizontal spacing comparable to their size. Lidar data show GW vertical displacements of $\pm 0.3 \mathrm{~km}$. From the data, we find a $\mathrm{GW}$ intrinsic frequency and vertical wavelength of $0.009 \pm 0.003 \mathrm{rad} \mathrm{s}^{-1}$ and $9 \pm 4 \mathrm{~km}$, respectively. We show that these values are consistent with the predictions of numerical simulations of idealized GW breaking. We estimate the momentum deposition rate per unit mass during this event to be $0.04 \pm 0.02 \mathrm{~m} \mathrm{~s}^{-}$ 2 and show that this value is consistent with the observed GW. Comparison to simulation gives a mean energy dissipation rate for this event of $0.05-0.4 \mathrm{~W} \mathrm{~kg}^{-1}$, which is consistent with other reported in-situ measurements at the Arctic summer mesopause.
\end{abstract}

\title{
1. Introduction
}

Our understanding of the multiple roles of gravity waves (GWs) generated in the lower atmosphere and propagating into the mesosphere and lower thermosphere (MLT) has advanced considerably since their identification in ionospheric irregularities and polar mesospheric clouds (PMCs) 60 years ago (Hines, 1960; Witt, 1962). We now know that GWs account for the major transports of energy and momentum from their various sources in the lower atmosphere into the MLT, and that diverse GW influences increase strongly with altitude due to decreasing density and increasing GW amplitudes. On global scales, mean GW energy dissipation and momentum deposition lead to systematic forcing of the zonal-mean circulation and thermal structure, and to accompanying induced residual circulations, at altitudes from the troposphere into the thermosphere (Fritts \& Alexander, 2003, and references therein).

At smaller scales, observations and modeling have revealed that local accelerations due to GW dissipation, or due to GW transience prior to dissipation, generate secondary GWs that can propagate to much higher altitudes (Bossert et al., 2017; Dong et al., 2019; Fritts, Dong, et al., 2019; Kaifler et al., 2017; Lane \& Sharman, 2006; Satomura \& Sato, 1999; Vadas et al., 2018). GW breaking also induces significant GW amplitude reductions that imply highly variable GW momentum fluxes and forcing in space and time (Fritts et al., 2009a, 2009b; Taylor et al. 2019). Limited observations also appear to confirm that GWs having smaller horizontal wavelengths, $\lambda_{h} \sim 100 \mathrm{~km}$ or smaller, account for the major momentum fluxes in the MLT (Fritts et al., 2002, 2014, 2018; Taylor et al., 2019).

The GW scales and dynamics accounting for the majority of these effects throughout the atmosphere are poorly quantified by sparse and sporadic observations. Similarly, GWs and their instabilities leading to turbulence also contribute to transport and mixing from the surface to altitudes above $100 \mathrm{~km}$, but the dependence of these responses on $\mathrm{GW}$ and instability dynamics is also poorly constrained by observations and modeling (Garcia et al., 2014). Hence, descriptions of GW influences in global climate and weather prediction models rely on simplifying assumptions that cannot account for known GW character and influences revealed by high-resolution observations and modeling (Geller et al., 2013; Kim et al., 2003).

GWs exhibit a wide range of instabilities, depending on their characters and environments. At small amplitudes, GWs exhibit resonant and non-resonant interactions that excite other GWs and broaden the GW spectrum without dissipation (McComas \& Bretherton, 1977; Sonmor \& Klaasen, 1997). Inertia-GWs at sufficiently large amplitudes at intrinsic frequencies near the 
inertial frequency, $\omega_{i} \sim f$, support Kelvin-Helmholtz instabilities (LeLong \& Dunkerton, 1998; Luce et al., 2008). GWs at higher frequencies more typically undergo breaking at amplitudes approaching or exceeding overturning conditions (Andreassen et al., 1994; Fritts et al., 1994), giving rise to convective instabilities and leading to the formation of vortex rings (Andreassen et al., 1998; Fritts et al., 1998; Fritts et al. 2009a, 2009b). These instabilities often arise from initial optimal perturbations to the evolving flows at GW amplitudes that are not overturning (Achatz, 2005, 2007; Lombard \& Riley, 1996). Additionally, multi-scale GW environments exhibit variants of the above instabilities, many of which have been observed by radar, lidar, and/or high-resolution imaging (Baumgarten \& Fritts, 2014; Eaton et al., 1995; Fritts et al., 2013; Fritts, Miller, et al., 2019; Lehmacher et al., 2007; Miller et al., 2015; Pfrommer et al., 2009). The character, importance, and effects of various GW instability dynamics for a range of relevant flows need to be explored and understood more completely if we are to account more quantitatively for their influences throughout the atmosphere.

In this paper we focus on vortex rings associated with GW convective instabilities. Witt (1962) was the first to report on observed small-scale holes in PMC images. The first identification of vortex rings in PMC images was by Dalin et al. (2010); further observations by ground-based (Fritts et al., 2017; Hecht et al., 2018) and balloon-based instruments (Fritts et al., 2017; Miller et al., 2015) followed. High-resolution numerical simulation by Fritts et al. (2017) described the three-dimensional (3-D) structure of these instabilities: counter-rotating vortices aligned along the direction of GW propagation intensify with increasing $\mathrm{GW}$ amplitude. Interactions between adjacent vortices lead to horseshoe-shaped vortices and eventually distinct vortex rings. These rings are inclined at $\sim 45^{\circ}$ relative to the horizontal, and cause plunging motions along their axes, down and in the direction of GW propagation. Fritts et al. (2017) also generated synthetic images of vortex ring formation at the PMC layer; see in particular Figure 11(d-e) of that paper. They demonstrated that vortex rings can produce circular features in the PMCs, though the appearance of those features depends on the altitude at which GW breaking occurs relative to the PMC layer - if GW breaking is offset from the PMC layer, only partial rings will form. Another signature of vortex rings in the synthetic PMC images are the trailing remnants of the original counter-rotating vortices. These trailing vortices link adjacent rings, resulting in a concentration of brightness at that linkage. Observations to date have consisted of 2-D images of this inherently 3-D process.

Studying instabilities associated with GWs at the mesopause is of particular interest, because at these altitudes the dynamics are dominated by wave and wave breaking influences. This is demonstrated by the large departures in the thermal structure of the MLT from radiative equilibrium, which can reach up to $50 \mathrm{~K}$. These departures are due to a meridional circulation from the summer to winter pole that is caused by GW drag at the mesopause; the magnitude of these departures implies persistent body forcing due to GW breaking of approximately $100 \mathrm{~m}$ $\mathrm{s}^{-1}$ day $^{-1}$ (Vincent, 2015). Thus, instability structures observed at these altitudes can be attributed with high confidence to GW breaking, particularly when the timescales involved are close to the buoyancy period.

We report on an observation of instability structures that we identify as vortex rings accompanying apparent GW breaking seen in the PMC layer at $\sim 82 \mathrm{~km}$. Images with high spatial and temporal resolution were collected by the PMC Turbo long-duration balloon-borne experiment (see the mission overview by Fritts, Miller, et al., 2019). The experiment also hosted a Rayleigh lidar that provided coincident data on the vertical structure of the PMC layer. With this 3-D information about the structure of the PMC layer and of the GW and instability structures, we characterized the underlying GW and compare the dynamical behavior to results from numerical simulations of Fritts et al. (2017) and Fritts, Wang, et al. (2019).

We provide an overview of the PMC Turbo experiment and the GW breaking event in Section 2. In Sections 3 and 4, we discuss the analysis of the imagery and lidar data that led to 
identification and characterization of the dominant GW accounting for the observed vortex rings. In Section 5 we summarize our findings and present our conclusions.

\section{PMC Turbo Instrumentation and Data}

PMC Turbo was a balloon-borne payload designed to study GW and instability dynamics near the mesopause. Instrumentation consisted of four wide-field and three narrow-field CCDbased cameras and a Rayleigh lidar. The wide-field cameras gave a combined field of view (FOV) spanning $100 \times 100 \mathrm{~km}$ with an average spatial resolution of $20 \mathrm{~m}$ per pixel when projected to the PMC layer. The narrow-field cameras each spanned a FOV of $10 \times 15 \mathrm{~km}$, were located at the regions of overlap between the wide-field cameras and had a spatial resolution of 3-4 m per pixel at the PMC layer. The Rayleigh lidar measured atmospheric backscatter along a line of sight that was $28^{\circ}$ off-zenith and within the FOV of the cameras. The arrival time of each photon was recorded, and binning was performed in post-processing. For the event analyzed here, a vertical resolution of $60 \mathrm{~m}$ and a temporal resolution of $5 \mathrm{~s}$ was used in quantifying the volume backscatter coefficient of the PMCs. For more details on the design and performance of the lidar system, see Kaifler et al (2020).

PMC Turbo flew for 5.9 days in July of 2018 from Kiruna, Sweden to Nunavut, Canada, approximately tracking the Arctic Circle. For an overview of the instrumentation, image processing, underlying weather, and a list and examples of the range of instability dynamics observed during flight, see Fritts, Miller, et al. (2019) and Kjellstrand et al. (2020).

PMCs were visible for $50 \%$ of flight, and instability structures were prevalent whenever PMCs were present. Vortex rings were one of the more common instability structures observed in the images. In this paper we discuss one particularly active period between 2:40 and 3:00 UT on 10 July 2018 during which vortex rings were present across much of the FOV. At these times, the gondola was floating at an altitude of $36.3 \mathrm{~km}$, at $69.3^{\circ} \mathrm{N}$ and $17.4^{\circ} \mathrm{W}$, about 200 miles north of Iceland over an intense tropospheric jet stream along the east coast of Greenland. The gondola was oriented such that the FOV was centered on the anti-sun direction, viewing approximately toward south-southwest. The gondola was drifting to the west-southwest at an average speed of $8 \mathrm{~m} \mathrm{~s}^{-1}$.

Representative PMC Turbo imaging and lidar data for this interval are shown in Figure 1. Panel (a) shows one of a total of 600 images of the field used in this analysis; panel (b) is an enlargement of a $20 \mathrm{~km}$ x $20 \mathrm{~km}$ section. Vortex rings are visible in the images as dark circular or slightly elliptical cores with diameters between 2 and $5 \mathrm{~km}$. The dark cores are surrounded by brighter PMCs, which in many cases are brighter than the image average. Examples are marked by arrows in panel (b). The vortex rings are distributed over an area of at least $60 \times 60$ $\mathrm{km}$. In Section 3.1 we provide evidence that the periodic darker and lighter regions in panel (a), oriented approximately SW-NE with an approximate wavelength of $20 \mathrm{~km}$, are a GW. The lidar data, in panel (c), show a bright PMC layer with average max brightness of $18 \times 10^{-10} / \mathrm{m} / \mathrm{sr}$ between 81 and $83 \mathrm{~km}$ in altitude. A temporal vertical oscillation with period of $\sim 10$ minutes is apparent, as well as a quick succession of downward features between 2:41 and 2:47 UT. We will present evidence linking the vertical oscillation to the underlying GW (Section 3.2) and the downward features to the passage of vortex rings (Section 4.4).

\section{Characterization of Gravity Wave and Local Winds}

Given the abundance of instability structures visible in the images, we infer that at least one GW was in the process of breaking at or near the PMC layer. In this section: we identify a GW in the image data and derive estimates for its horizontal wavelength and apparent phase speed; we use the lidar data to cross check the conclusions of the image analysis; and we use images and independent wind data to constrain the mean winds and therefore the GW's intrinsic phase speed. 
We search for a GW in the image data because there are at least two mechanisms by which it can imprint its signature on PMC brightness: (1) GW-induced velocity perturbations cause regions of horizontal convergence and divergence at the PMC layer. The imaging data are only sensitive to the integrated brightness through the PMC layer, therefore vertical displacements associated with the velocity perturbations are irrelevant to this effect. In areas of horizontal convergence, there is greater column density of PMC particles, leading to higher brightness in the images; (2) the GW induces vertical perturbations of the PMC layer, causing associated temperature perturbations. Relatively warm regions associated with downward perturbations act to sublimate PMC particles, decreasing brightness, while cool regions (upward perturbations) enhance brightness by facilitating PMC particle formation and growth, though increased water vapor concentrations at lower altitudes may increase brightness, counteracting this effect. Estimates vary over the range of possible growth and sublimation rates of PMC particles (Chandran et al., 2012; Gadsden and Schröder, 1989; Rapp et al., 2002; Zasetsky et al., 2009); it is inconclusive whether this effect can be significant for dynamics that evolve on timescales on the order of minutes. Given the spectral response of our instruments $(\geq 600 \mathrm{~nm}$ for the cameras and $532 \mathrm{~nm}$ for the lidar), we approximate our observed signal as Rayleigh scattering. Given the implied $r^{6}\left(r^{5.5}\right.$, see Savigny \& Hoffman, 2020) dependence of brightness on particle size $r$, a $5 \%$ change in particle size would result in a $34 \%(30 \%)$ change in PMC brightness, a magnitude detectable in the image data. Assuming a typical particle size of $55 \mathrm{~nm}$ (Ugolnikov et al., 2016), this corresponds to a change of $2.8 \mathrm{~nm}$; this change can occur within 5 min, given the upper range of the growth rates measured in Zasetsky et al. (2009). Thus, for the timescales discussed here, particle growth and sublimation driven by temperature perturbations are a plausible mechanism for PMC brightness modulation.

The flat-fielding process applied to the raw images includes subtraction of a 10 min moving window and therefore removes absolute PMC brightness information (Fritts, Miller, et al., 2019). The processed images display relative brightness in uncalibrated counts normalized for exposure time and pixel sensitivity. Raw images were acquired in groups of 2-4 at a cadence of $2 \mathrm{~s}$; for this analysis, we used images at either a 2 or a $10 \mathrm{~s}$ cadence. The coordinate system we use has the $\mathrm{y}$-axis oriented northward and the $\mathrm{x}$-axis eastward. Images are displayed as viewed from above and are projected onto the PMC layer, assumed to be a plane at an altitude of $82 \mathrm{~km}$. The coordinate system is fixed relative to the Earth such that the origin was directly above the gondola at 2:50 UT on 10 July 2018. Due to insufficient shading between the sunlit balloon and the cameras, images were often contaminated by scattered light. We report results using only the two central wide-field cameras, which were least affected.

\subsection{GW propagation direction, horizontal wavelength, and apparent phase speed}

In order to characterize the dominant $\mathrm{GW}$, we searched for an orientation at which the brightness modulated periodically across the FOV, in the following way. We divided the images into $1 \mathrm{~km}$-wide strips, averaged the brightness in each strip, and plotted the average brightness as a function of distance along the direction perpendicular to the strips. The analysis was repeated at varying orientations of the strips, in $5^{\circ}$ intervals. When the orientation of the strips aligned with the phase lines of the underlying $\mathrm{GW}$, we expected the average brightness of the strips to vary sinusoidally across the image. The orientation of the GW was determined by finding the best-fit sinusoid for each of these averages, allowing amplitude, frequency, phase and vertical offset to vary, and then plotting the amplitude of the sinusoid as a function of angle. We found the angle at which the amplitude of the fit was largest and assumed that at that angle the averaging was along a common phase of the GW. This maximum occurred at $35^{\circ}$ clockwise from north, corresponding to a GW propagation direction, $\hat{k}$, of $125^{\circ}$ clockwise from north. Examples of these averages together with their sinusoidal fits are shown in Figure 2. We define streamwise to be the direction of $\mathrm{GW}$ propagation, and spanwise to be 
perpendicular to streamwise. The streamwise distance is defined in the fixed coordinate system described in Section 3 such that points along a common phase with the origin are defined to have a streamwise position of $0 \mathrm{~km}$.

Quantifying the apparent phase propagation is complicated by the evolution of the phase structure with time, due to the presence of bright small scale structures and variation in the local winds across the field of view (see Figure 8). To estimate the phase propagation, we took sets of images at $10 \mathrm{~s}$ intervals and plotted the spanwise average across these images as a function of distance in the streamwise direction, assuming the determined GW orientation. Periodic structure is clearly visible in each plot, and phase propagation is apparent in the sequence; see Figure 3. We found the best-fit location of each peak and trough at each time interval by chi-squared fitting a Gaussian over a range of half a wavelength in the vicinity of each peak/trough, allowing the location, amplitude, width, and vertical offset to vary (see Figure 4). For each time interval, we found the average separation of adjacent peaks/troughs. Averaging across all time intervals gave an estimate of the horizontal wavelength, with $\lambda_{h}=$ $18 \pm 0.5 \mathrm{~km}$, where the quoted uncertainty reflects the variance among the measurements.

We performed a linear fit of the position of each peak and trough as a function of time, giving apparent phase speed for each peak and trough (see Figure 4). We took the slope of each fit as a measurement of the apparent phase speed and found an average value of $c=21 \pm 6$ $\mathrm{m} \mathrm{s}^{-1}$, where the quoted uncertainty indicates the variance between the slopes - the uncertainty in the motion of an individual peak/trough is less than $1 \mathrm{~m} \mathrm{~s}^{-1}$. We also performed a 2-D Fourier transform on the image data after interpolating onto a regularly-spaced grid sampled at $20 \mathrm{~m}$ in either direction (see Figure 3). This confirmed the orientation and horizontal wavelength found above but had higher associated uncertainties: $\lambda_{h}=17 \pm 1.7 \mathrm{~km}$ and a propagation direction of $123^{\circ} \pm 6^{\circ}$.

\subsection{Identification of $\mathbf{G W}$ in lidar data}

We use the independent lidar data to confirm the presence of the GW identified above. We find the signature of the GW in the altitude of the PMC layer as measured by the lidar, and we compare this signal to the spanwise-averaged brightness of the image data at the location of the lidar beam.

\subsubsection{Vertical perturbations of the PMC altitude}

The lidar data show an overall vertical oscillation with a period of $\sim 600 \mathrm{~s}$. In order to quantify this motion further, we estimated the altitude of the PMC layer by finding the altitude of maximum PMC brightness at all times. We found the amplitude of the best-fit sinusoid to this altitude to be $0.27 \pm 0.05 \mathrm{~km}$. The calculated PMC altitude and sinusoidal fit are shown in red and black, respectively, in the bottom plot of Figure 5.

\subsubsection{GW brightness perturbations at the lidar beam}

To compare the results of our imaging analysis to the lidar profiles, we determined the location within our camera FOV where the lidar beam intersected with the cloud layer. The FOV of the lidar was approximately the same as that of a single pixel. We solved for the relative alignment by generating two datasets: 1) the integrated PMC brightness from the lidar profile, and 2) time series of flat-fielded camera brightness on a pixel-by-pixel basis over a $75 \times 75$ pixel grid in the nominal neighborhood of the lidar beam. We integrated the camera data over $10 \mathrm{~s}$ intervals to match the lidar cadence and removed a 10 min moving average from the lidar time series to match the camera flat-fielding process. We then computed the Pearson p-value of the lidar time series with each of the camera time series. We determined the location of overlap between the instruments by fitting a 2-D Gaussian profile to the logarithm of the p-value over the $75 \times 75$ pixel grid. A $\chi^{2}$ test gives the best-fit location of the overlap between the lidar and 
the camera FOVs; the uncertainty in this process is \pm 3 pixels in either direction, or $\pm 20 \mathrm{~m}$ on the PMC layer. The normalized time series of the lidar and the camera pixel at the location of overlap are shown in the top plot in Figure 5.

To identify the GW phase at the location of the lidar beam, we again took the spanwise average of the full image data at each time step in the lidar data and found the value at the streamwise location of the lidar. We subtracted an offset and a linear trend to help isolate the GW signal. The result is shown in blue in the bottom plot in Figure 5.

\subsubsection{Comparison of GW signal in lidar and image data}

Comparing the PMC layer altitude to the spanwise-averaged image brightness (see Figure 5), the periodic structure is apparent in each and is strongly correlated. The lidar therefore detects a GW signal that is consistent with the GW identified in the camera data.

The two signals are closely aligned in phase. Recalling the mechanisms by which a GW can imprint its signature on PMC brightness (Section 3): if the increased brightness is due to horizontal convergence of the PMC particles, we expect this to occur downstream (i.e. positive streamwise distance) from the maximum vertical displacement. The phase shift between these quantities is $90^{\circ}$ at small $\mathrm{GW}$ amplitudes, but approaches $0^{\circ}$ as the GW approaches its overturning amplitude Conversely, if the temperature mechanism is responsible, the rate of change in brightness will be in phase with the vertical displacements, but the maximum integrated change in brightness will occur upstream (negative streamwise distance) from the maximum vertical displacement. Again, at small amplitudes the phase shift is expected to be $90^{\circ}$; for GWs close to overturning, this phase shift will increase to $180^{\circ}$. Given that the lidar is effectively scanning in the upstream direction as the GW propagates through the lidar beam, the GW brightness signal is slightly downstream relative to the vertical displacements. This suggests that the observed brightness is due primarily to the horizontal convergence of PMC particles rather than PMC particle growth and sublimation in response to GW temperature perturbations; additionally, the small phase shift between the GW brightness and altitude is consistent with a GW that is in the process of breaking.

\subsection{Characterization of the local mean winds}

The intrinsic phase speed and frequency of the GW identified above depend on the mean winds in which the GW propagated. We obtained two independent estimates of the mean winds to quantify these additional GW properties.

\subsubsection{Wind estimates from NAVGEM Reanalysis}

We obtained an estimate of the local large-scale winds and temperatures from the reanalysis fields of the U.S. Navy Global Environmental Model (NAVGEM: Eckermann et al., 2018), generated with a T119L74 forecast model and hybrid 4-D variational data assimilation procedure that as of recently includes measured winds at altitudes between 80 and $100 \mathrm{~km}$ from 25 meteor-radar sites around the world. Given the $100 \mathrm{~km}$ horizontal resolution of the reanalysis results, we expect it to resolve GWs with $\lambda_{h}>500 \mathrm{~km}$; therefore, it is insensitive to the GW we have observed here but should give estimates of the broader background in which the GW breaking occurred. The resulting wind estimates vs. altitude, taken over an averaging radius of $200 \mathrm{~km}$ horizontally around the location of the gondola, at 2:00 UT and at 3:00 UT, are shown in Figure 6. We infer a mean wind vector $\vec{U}_{0}=\left(\begin{array}{l}-28 \pm 9 \\ -29 \pm 4\end{array}\right) \mathrm{m} \mathrm{s}^{-1}$; the x-component is the zonal direction. Uncertainties come from a comparison of NAVGEM predictions to measured winds at $82 \mathrm{~km}$ (see Fig. 15 of Eckermann et al., 2018).

NAVGEM also provides estimates of the background temperature and buoyancy frequency $N$ as a function of altitude. We estimate $N=0.021 \pm 0.004 \mathrm{rad} \mathrm{s}^{-1}$, where the inferred uncertainty comes from the steep change in $N$ with altitude; see Figure 6 . We use the estimate 
of the background temperature to calculate the atmospheric scale height, $H=\frac{R T_{0}}{M g}$, where $R$ is the universal gas constant, $T_{0}$ is the background temperature, $M$ is the mean molecular molar mass, and $g$ is the acceleration due to gravity, giving $H=4.4 \pm 0.1 \mathrm{~km}$. Note also that GWs unresolved by NAVGEM, such as the GW identified here, likely also contributed to local $N(z)$, temperatures, and winds.

\subsubsection{Wind estimates from Trackpy feature tracking}

Bright and ubiquitous instability structures across the FOV enabled the use of feature tracking to characterize the mean winds at the PMC layer. We used Trackpy (Trackpy v04.1, 2019), an open source python package that finds features in images and links such features in sequences of images to estimate motions. We used images at a $2 \mathrm{~s}$ cadence to reduce motion between consecutive frames and increase the probability that features are correctly linked.

To gain confidence that Trackpy identified reasonable features, linked them correctly in series of images, and reported accurate and robust estimates of the background flow, we performed a series of tests, which we summarize here. We

- ran Trackpy on simulated data with known input velocity between images;

- manually tracked several features in the camera images to validate Trackpy results;

- spot-checked individual Trackpy trajectories against image data to confirm that Trackpy features appear to advect with visible structure in the images (see Figure 7);

- tested the robustness of the results against a range of input parameters to the Trackpy algorithm. These parameters included: the characteristic feature size (11-400 pixels), the minimum integrated brightness of a given feature (we found no correlation between minimum brightness and inferred velocities.), and the minimum number of occurrences a feature must make (10-50 occurrences);

- ran Trackpy on simulated data with GW and vortex ring structures propagating differentially. The relative amplitudes of these signals were derived from the image data, as was the background noise level.

In all cases, Trackpy gave reasonable results. In particular, in the final test we determined that the inferred velocities were those of the vortex rings and were not affected by the presence or motion of the GW. The advantage of Trackpy is that it found many more features than would be feasible to track manually, which provided information on trends in the winds across the field of view rather than a single average value.

Due to the 10 min moving average that we subtracted in our flat-fielding process, stars in our field of view produced dark tracks $\sim 5 \mathrm{~km}$ long in the flat-fielded images, with a bright spot in the middle of the track corresponding to the current position of the star. Therefore, we ran a low-pass Gaussian 2-D filter to remove features smaller than 31 pixels before passing the images to Trackpy. We then used $8 \times 8$ pixel binning to improve the signal to noise within our images. Our tests determined that the optimal characteristic feature size was 7 pixels (corresponding to 56 pixels in native pixel dimensions), or $\sim 1 \mathrm{~km}$ when projected onto the sky. A typical vortex ring has a diameter of $\sim 2-5 \mathrm{~km}$ (see the example image shown Figure 1, which is representative). A feature therefore corresponds to a section of a vortex ring rather than an entire ring, or to some smaller instability structure.

For the 20 min duration of the interval analyzed here, Trackpy found 5,004 'trajectories'. A trajectory corresponds to a single feature that Trackpy found repeatedly across many images. For each trajectory, Trackpy output a list of positions in pixel coordinates and corresponding images, which we converted to a list of positions at the PMC layer and corresponding times. For each trajectory, we assumed a constant velocity and performed a linear fit in the meridional and zonal directions independently, resulting in an estimate for the meridional and zonal winds for this feature. We filtered out trajectories with a probability-to-exceed of $<0.5 \%$ according to 
the $\chi^{2}$ metric in either the zonal or meridional fit, resulting in 3,916 remaining trajectories. A subset of these remaining trajectories and the corresponding linear fits are shown in the top plot of Figure 7 . We averaged the best-fit winds across all the trajectories and found mean winds, denoted $\vec{U}_{\text {local }}$ to differentiate from the NAVGEM-derived $\vec{U}_{0}$, of $\left(\begin{array}{l}-10 \pm 5 \\ -50 \pm 9\end{array}\right) \mathrm{m} \mathrm{s}^{-1}$. Given the large number of trajectories, the uncertainty on the mean is negligible - instead, the quoted uncertainties indicate the standard deviation of the inferred velocities and is mainly driven by variation across the FOV (see Figure 8). These values differ significantly from those obtained from the NAVGEM reanalysis (Section 3.3.1); we discuss this discrepancy in Section 4.2.

\section{Discussion}

\subsection{Estimates of GW Parameters}

From our analysis above, we have inferred estimates of $\lambda_{h}$ and $c$. Given the inferred propagation direction and estimates of the background environment provided by NAVGEM, we draw further conclusions about the parameters of the underlying GW, using standard relationships derived from linear GW theory (see, for example Nappo, 2002) and assuming a monochromatic GW:

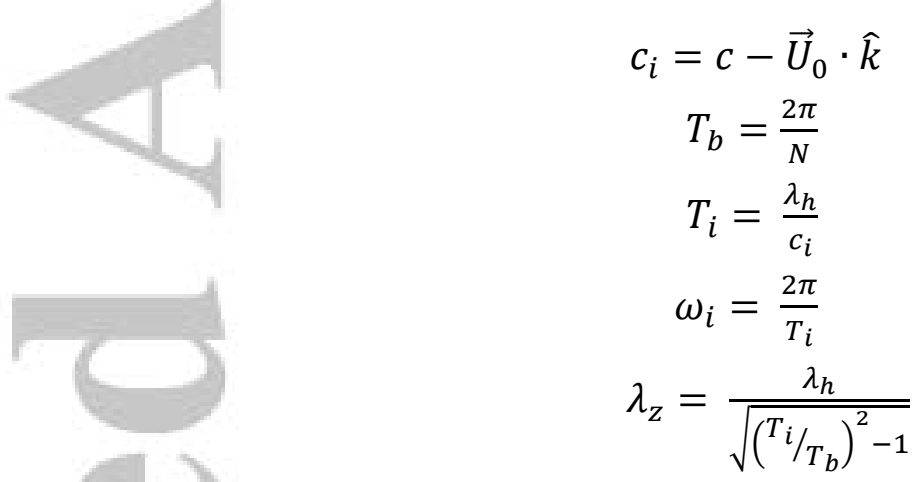

Here, $c_{i}$ is the intrinsic phase speed of the $\mathrm{GW}$, and $T_{i}, \omega_{i}$ and $\lambda_{z}$ are its period, intrinsic frequency and vertical wavelength, respectively. $T_{b}$ is the buoyancy period. Given $\hat{k}, \vec{U}_{0}$, $\lambda_{h}$ and $c$ as determined previously, we infer the parameters listed in Table 1 . The uncertainties listed for derived quantities come from standard uncertainty propagation. Note that we use the estimates of the mean winds from NAVGEM rather than Trackpy - our motivation for this choice is given in Section 4.2.

The lidar profile provides an independent check on the vertical wavelength, though only as a lower-bound. We define the dimensionless GW amplitude to be $A=u_{1} / c_{i}$, where $u_{1}$ is the horizontal velocity perturbations induced by the GW. For a GW at its overturning amplitude $(A \approx 1)$, we expect vertical displacement amplitudes on the order of $\lambda_{z} / 2 \pi$ (see, for example, Fritts, Miller, et al., 2019). From the sinusoidal fit to the PMC layer centroid altitude, shown in Figure 5, we infer a lower bound of $\lambda_{z} \geq 1.7 \mathrm{~km}$. This is well below the value in Table 1 . Two factors may account for this. First, the presence of vortex rings implies that the GW had undergone significant breaking, which would act to reduce the GW amplitude and therefore the vertical perturbations to the PMC layer. Secondly, large vertical displacements are accompanied by large temperature perturbations. PMC particles advected to significantly warmer temperatures may encounter rapid sublimation, reducing the apparent amplitude of the vertical displacement visible in the PMC layer. 


\subsection{Estimate of GW momentum deposition}

The difference between the Trackpy and NAVGEM estimates of the mean winds is $\left(\begin{array}{c}18 \pm 10 \\ -21 \pm 10\end{array}\right) \mathrm{m} \mathrm{s}^{-1}$, an apparent $>2 \sigma$ discrepancy. We explain this apparent discrepancy in the following way. The abundance of vortex rings implies that the GW, initially at or close to the overturning amplitude, had undergone significant breaking. In the process, GW momentum flux divergence led to local flow accelerations along the direction of GW propagation therefore, we expect that local estimates of wind speeds will differ from large scale winds, such as those inferred by NAVGEM.

We quantify the expected magnitude of the momentum deposition as follows. A GW with velocity perturbations $u_{1}$ and $w_{1}$ (horizontally and vertically) results in a vertical flux of horizontal momentum, $\tau$, given by:

$$
\tau=-\rho_{0} \overline{u_{1} w_{1}} \approx \frac{1}{2} \rho_{0} \frac{k}{m} u_{1}^{2}
$$

where $\rho_{0}$ is the unperturbed background density, $k$ and $m$ are the horizontal and vertical wavenumbers, respectively, and the bar indicates a horizontal average over a wavelength. We have used the simplifying assumption (borne out by the data in Table 1) that $m$ is large relative to $1 / 2 H$. When such a GW breaks, its momentum flux decreases quadratically with decreasing amplitude, and the mean accelerations that accompanied GW propagation prior to breaking result in a transient mean-flow forcing. Numerical simulations reported in Fritts et al. (2009a) suggest a typical timescale for GW breaking of approximately $T_{i}$. We approximate the vertical length scale over which breaking occurs to be $H$. We expect a net change in the local momentum density given by:

$$
\rho_{0} \Delta u \sim \frac{T_{i}}{H} \tau
$$

which, together with Equation 4.2.1, implies a net change in local wind speeds of:

$$
\Delta u \sim \frac{1}{2 H} \frac{k u_{1}^{2} T_{i}}{m}
$$

Given that the GW broke, we infer that it had reached the overturning amplitude; thus $u_{1} \approx$ $c_{i}$, and $u_{1} T_{i} \approx \lambda_{h}$, implying

$$
\Delta u \sim \frac{1}{2 H} \frac{k \lambda_{h}}{m} c_{i} \sim \frac{\lambda_{z}}{2 H} c_{i}
$$

Lindzen (1981) derived the acceleration induced by a breaking GW by positing that above some critical altitude, $z_{\text {break }}$, diffusive turbulence will prevent further growth of GW amplitude, leading to momentum deposition at altitudes above $z_{\text {break }}$. Imposing this condition and examining the vertical derivative of $\tau$, he found the following expression for the acceleration, $a$, of the local mean flow:

$$
a=\frac{k}{2 H} \frac{c_{i}^{3}}{N}
$$

Here, we have converted Lindzen's expression to match the conventions used in this paper. Lindzen was interested in the acceleration in the zonal direction of a GW propagating in an arbitrary direction, but for the sake of comparison we generalize Lindzen's result to give the acceleration in the direction of GW propagation. Assuming that the acceleration persists over one period (the same assumption made in our derivation above), the net change in velocity due to GW breaking is given by:

$$
\Delta u=a T_{i}=\frac{k}{2 H} \frac{\lambda_{h} c_{i}^{2}}{N}=\frac{1}{2 H} \frac{\omega_{i}}{N} \lambda_{h} c_{i}
$$


where we have used the expressions $T_{i}=\lambda_{h} / c_{i}$ and $\omega_{i}=k c_{i}$. With the further approximation that $\omega_{i} /{ }_{N}=k / m$ (which holds generally for hydrostatic high-frequency GWs and is accurate to $10 \%$ in our case), then we find that

$$
\Delta u=\frac{\lambda_{h}}{2 H} \frac{k}{m} c_{i}=\frac{\lambda_{z}}{2 H} c_{i}
$$

which agrees with our result.

Thus, we expect a net change in the local winds of $\Delta u=\left(\begin{array}{c}23 \pm 12 \\ -16 \pm 8\end{array}\right) \mathrm{m} \mathrm{s}^{-1}$ relative to the background winds, implying local winds of $\vec{U}_{0}+\Delta u=\left(\begin{array}{c}5 \pm 15 \\ -45 \pm 9\end{array}\right) \mathrm{m} \mathrm{s}^{-1}$, which agrees with $\vec{U}_{\text {local }}$, as derived in Section 3.3.2; thus, the apparent discrepancy between the Trackpy and NAVGEM estimates for background wind speed is therefore consistent, both in magnitude and in direction, with local momentum deposition by the GW as it transitioned through instabilities to turbulence. Converting the net change in local winds, $\Delta u$, to a momentum deposition rate per unit mass, we find $\Delta u / T_{i}=0.04 \pm 0.02 \mathrm{~m} \mathrm{~s}^{-2}$.

As a further check, we evaluated the wind speeds at $2 \mathrm{UT}, \sim 30 \mathrm{~min}$ before the onset of strong GW breaking, using Trackpy and NAVGEM separately. Here, Trackpy gave an estimate of $\left(\begin{array}{c}-17 \pm 8 \\ -37 \pm 11\end{array}\right) \mathrm{m} \mathrm{s}^{-1}$ and NAVGEM gave an estimate of $\left(\begin{array}{l}-16 \pm 9 \\ -26 \pm 4\end{array}\right) \mathrm{m} \mathrm{s}^{-1}$. The two results are consistent with each other prior to the onset of GW breaking; this supports the hypothesis that the discrepancy at later times is due to local momentum deposition by the GW and not, for example, some systematic error in the NAVGEM or Trackpy-derived results.

We further examined the variation of the Trackpy-inferred winds as a function of position within the field of view. We assigned each Trackpy trajectory a location in the streamwise direction corresponding to its average location. We then examined the inferred streamwise and spanwise winds as a function of distance in the streamwise direction; see Figure 8. Given the presence of a GW, we expected to find phase-synchronous velocity perturbations in the streamwise direction. Such a signal did not appear.

To impose an upper limit on the magnitude of any phase-synchronous perturbation in the streamwise velocity, we removed a linear trend from the streamwise velocity data as a function of distance in the streamwise direction. We then found the best-fit phase-synchronous signal given the known GW wavelength using $\chi^{2}$ minimization. The amplitude of this fit was consistent with zero and had an upper limit of $2.2 \mathrm{~m} \mathrm{~s}^{-1}$. Assuming the GW was initially near its overturning amplitude (prior to entering the FOV of the instrument), this implies a >90\% reduction in amplitude over the GW breaking process, supporting the hypothesis that significant momentum deposition had already occurred.

Evident in the velocity data is an overall increase in the streamwise component of the flow - i.e. a net acceleration in the direction of GW propagation. This can be explained by nonuniform momentum deposition, a product of the spatial variability of the GW amplitude.

\subsection{Comparison to numerical simulations: vortex ring diameters, separations, lifetimes, and energy dissipation rate}

GW breaking leads to formation of vortex rings, a phenomenon that has been reproduced in simulations (Fritts et al., 1998; Fritts et al., 2009a; Fritts et al., 2017; Fritts, Wang, et al., 2019). The simulations suggest relationships between the characteristics of the vortex rings and the parameters of the underlying $\mathrm{GW}$. The observation reported here provides the first empirical test of these predicted relationships.

Simulations indicate that the diameters of the largest vortex rings scale with GW vertical wavelength: $D \sim \lambda_{z} / 2$, where $D$ is the largest vortex ring diameter where it is well-defined prior 
to breakup (Fritts et al., 2017). The vortex rings observed here range in size from $2-5 \mathrm{~km}$, implying a $\lambda_{z}$ of $\sim 10 \mathrm{~km}$, in agreement with our measurements (Table 1). Simulations indicate that vortex ring separations that are comparable to the vortex ring size (i.e. vortex rings that are close to overlapping) indicate an intrinsic frequency $\omega_{i} \gtrsim N / 3$ (Fritts, Wang, et al., 2019). The vortex rings observed here are closely-spaced (see Figure 9), and the relationship we infer between $\omega_{i}$ and $N$ is consistent with simulations (Table 1).

Note that previous observations provide further confirmation: vortex rings observed in airglow images of breaking mountain waves over the southern Andes by Hecht et al. (2018) had diameters $(5 \mathrm{~km})$ that were broadly consistent with the inferred $\mathrm{GW}$ vertical wavelength (13-14 km) as predicted by Fritts et al. (2017).

Another point of comparison to the results of Fritts et al. (2017) is the expected vortex ring breakdown time. In the numerical simulations, the vortex rings were seen to evolve from a coherent ring to strong turbulence within $\sim 1 T_{b}$. The evolution shown in Fig. 9 illustrates the full life cycle of a pair of vortex rings with a total lifetime of $\sim 600 \mathrm{~s}$, or approximately $2 T_{b}$. The vortex rings first start to lose definition in panel (f), and by panel (j) all definition is lost an interval of $320 \mathrm{~s}$, or approximately $1 T_{b}$. Thus, the timescales observed here match those predicted by Fritts et al. (2017).

Fritts et al. (2017) found in simulations that the energy dissipation rate varied by several orders of magnitude both spatially and temporally. They characterize the energy dissipation rate by the mean and peak energy dissipation rates: $\langle\varepsilon\rangle$ and $\varepsilon_{\text {high }}$, respectively, where the peak rate is defined to be the $99^{\text {th }}$ percentile value over the domain. They defined a scaling parameter $C=\lambda_{z}^{2} / T_{b}^{3}$. With PMC Turbo data, we find $C=2.7_{-2.2}^{+2.7} \mathrm{~m}^{2} \mathrm{~s}^{-3}$. From the unscaled estimates given in Fritts et al. (2017), we infer $\langle\varepsilon\rangle$ between $0.05 \mathrm{~W} \mathrm{~kg}^{-1}$ and $0.4 \mathrm{~W} \mathrm{~kg}^{-1}$, and $\varepsilon_{\text {high }}$ between $0.4 \mathrm{~W} \mathrm{~kg}^{-1}$ and $4.8 \mathrm{~W} \mathrm{~kg}^{-1}$. These values are consistent with in-situ assessments at the Arctic summer mesopause: e.g. Lübken et al. (2002) and Strelnikov et al. (2009) measured rates up to $2.4 \mathrm{~W} \mathrm{~kg}^{-1}$ and $2.0 \mathrm{~W} \mathrm{~kg}^{-1}$, respectively. As pointed out by Fritts et al. (2017), these in-situ measurements are very localized and thus unlikely to measure the peak energy dissipation rates seen in simulations.

\subsection{3-D structure of the vortex rings}

This is the first time vortex rings have been seen in lidar data; such identification is only possible through the coincidence of lidar and image data. In the lidar time series, the event is characterized by rapid downward excursions of the bottom of the PMC layer, spaced by 1-2 min (see red arrows in the bottom plot of Figure 10). The image data reveal the nature of these perturbations: small-scale instabilities with characteristic sizes of $\sim 2-5 \mathrm{~km}$ were advected through the lidar beam at a relative speed of $\sim 50 \mathrm{~m} \mathrm{~s}^{-1}$. From 2:48 UT onward, the vortex rings in the vicinity of the lidar beam are sparse and relatively weak; in this section of data, the lidar reveals a cloud layer that is roughly $1 \mathrm{~km}$ thick. This leads to insight into the 3 -D structure of the vortex rings: the bright, downward excursions are in fact relatively undisturbed regions of the PMC layer with the initial thickness of $\sim 1 \mathrm{~km}$. The gaps between these downward excursions correspond to the centers of the vortex rings. These observations agree with the simulations of Fritts et al. (2017), which show vortex rings that are inclined at $\sim 45^{\circ}$ to the horizontal and are characterized by plunging motions along their axes (down and in the streamwise direction). These plunging motions displace the cloud particles horizontally and accelerate sublimation due to the rapid adiabatic warming associated with downward motions. A movie showing the coincident lidar and imaging data is included in the supplementary materials (see S2.mp4); a few representative frames are shown in Figure 10, with blue arrows showing the corresponding time of each frame in the lidar data, and white circles marking the 
position of the vortex ring in each image; the circles are oversized to avoid obstructing the ring itself.

Quasi-periodic oscillations with frequencies higher than the expected buoyancy period have been observed in past mesospheric lidar data (see for example, Kaifler et al., 2018). These have been interpreted as acoustic waves or Doppler-shifted GWs. The coincident PMC Turbo imaging data demonstrates that past observations may in fact have been manifestations of instability dynamics.

\section{Conclusions}

We identified a prominent GW in PMC Turbo images that contain vortex rings with diameters of $2-5 \mathrm{~km}$. We hypothesized that this GW was responsible for the formation of the vortex rings. We used 600 images spanning 20 minutes and contemporaneous lidar data to determine the properties of the GW including propagation direction, phase speed, and horizontal wavelength. Together with estimates of the background conditions, the data provided evidence for an underlying GW with vertical wavelength of $\sim 9 \mathrm{~km}$ and intrinsic frequency $\sim 0.009 \mathrm{rad} \mathrm{s}^{-1}(N / 2.2)$. We explained the difference between the inferred mean local winds as obtained with two independent methods as a consequence of GW breaking and implied mean forcing. We showed that the difference is consistent, in magnitude and direction, with momentum deposition by a GW initially at the overturning amplitude, according to linear GW theory.

This is the first coincident observation in lidar and image data of PMC structures that are identified in image data as vortex rings. As such, it is the first confirmed observation of vortex rings in lidar data. The coincident data support the 3-D description of vortex rings seen in numerical simulations. Finally, we confirmed relationships between characteristics of the vortex rings and parameters of the underlying $\mathrm{GW}$, as derived from numerical simulations of GW breaking and previously uncorroborated by experiment, and we used the simulations to determine the range of energy dissipation rates relevant to this event.

Acknowledgements: We thank the paper reviewers for their valuable feedback. Research described here was supported under the NASA grant cited in GEMS. This project also received funding from the German Aerospace Center (DLR) for construction, integration, and operation of the Rayleigh lidar and subsequent data analyses. CG acknowledges support for this research from the University of Minnesota Graduate School and the Minnesota Space Grant Consortium. SDE acknowledges support for this research from the Chief of Naval Research via the base 6.1 program and from the DARPA Space Environment Exploitation (SEE) program. NAVGEM runs were made possible by a grant of computer time from the DoD High Performance Computing Modernization Program at the Navy DoD Supercomputing Resource Center. Links to data required to replicate all PMC Turbo images and other figures in this paper are available in the supporting documentation. 


\section{References}

Achatz, U. (2005). On the role of optimal perturbations in the instability of monochromatic gravity waves. Physics of Fluids, 17(9), 94-107. https://doi.org/10.1063/1.2046709

Achatz, U. (2007). The primary nonlinear dynamics of modal and nonmodal perturbations of monochromatic inertia-gravity waves. Journal of the Atmospheric Sciences, 64(1), 7495. https://doi.org/10.1175/JAS3827.1

Andreassen, Ø., Wasberg, C.-E., Fritts, D. C., \& Isler, J. R. (1994). Gravity wave breaking in two and three dimensions: 1. Model description and comparison of two-dimensional evolutions. Journal of Geophysical Research, 99, 80958108. https://doi.org/10.1029/93JD03435

Andreassen, Ø., Hvidsten, P.Ø., Fritts, D.C., \& Arendt, S. (1998). Vorticity dynamics in a breaking internal gravity wave: 1. Initial instability evolution. Journal of Fluid Dynamics, 367, 27-46. https://doi.org/10.1017/S0022112098001645

Baumgarten, G., \& Fritts, D. C. (2014). Quantifying Kelvin-Helmholtz instability dynamics observed in noctilucent clouds: 1. Methods and observations. Journal of Geophysical Research: Atmospheres, 119, 9324- 9337. https://doi.org/10.1002/2014JD021832

Bossert, K., Kruse, C.G., Heale, C.J., Fritts, D.C., Williams, B.P., Snively, J.B., et al. (2017). Secondary gravity wave generation over New Zealand during the DEEPWAVE campaign. Journal of Geophysical Research: Atmospheres, 122, 7834-7850.

https://doi.org/10.1002/2016JD026079

Chandran, A., Rusch, D.W., Thomas, G.E., Palo, S.E., Baumgarten, G., Jensen, E.J., \& Merkel, A.W. (2012). Atmospheric gravity wave effects on polar mesospheric clouds: A comparison of numerical simulations from CARMA 2D with AIM observations. Journal of Geophysical Research, 117, D20104. http://dx.doi.org/10.1029/2012JD017794

Dalin, P., Pertsev, N., Frandsen, S., Hansen, O., Andersen, H., Dubietis, A., \& Balciunas, R. (2010). A case study of the evolution of a Kelvin-Helmholtz wave and turbulence in noctilucent clouds. Journal of Atmospheric and Solar - Terrestrial Physics, 72(14-15), 11291138

https://doi.org/10.1016/j.jastp.2010.06.011

Dong, W., Fritts, D.C, Lund, T.S., Wieland, S.A., \& Zhang, S. (2019) Self-acceleration and instability of gravity wave packets: 2 . Two-dimensional packet propagation, instability dynamics, and transient flow responses. Journal of Geophysical Research: Atmospheres, 125(3). https://doi.org/10.1029/2019JD030691

Eaton, F., Mclaughlin, S. A., \& Hines, J. R. (1995). A new frequency modulated continuous wave radar for studying planetary boundary layer morphology. Radio Science, 30(1), 75-88.

https://doi.org/10.1029/94RS01937

Eckermann, S. D., Ma, J., Hoppel, K.W., Kuhl, D.D., Allen, D.R., Doyle, J.A., et al. (2018). High-altitude $(0-100 \mathrm{~km})$ global atmospheric reanalysis system: Description and application to 
the 2014 austral winter of the Deep Propagating Gravity-Wave Experiment (DEEPWAVE), Monthly Weather Review, 146, 2,639-2,666. https://doi.org/10.1175/MWR-D-17-0386.1

Fritts, D.C., \& Alexander, M. J. (2003). Gravity dynamics and effects in the middle atmosphere. Reviews of Geophysics, 41(1), 1003, https://doi.org/10.1029/2001RG000106

Fritts, D.C., Isler, J. R., \& Andreassen, Ø. (1994). Gravity wave breaking in two and three dimensions: 2. Three-dimensional evolution and instability structure. Journal of Geophysical Research, 99, 8,109-8,123, https://doi.org/10.1029/93GL01982

Fritts, D. C., Arendt, S., \& Andreassen, Ø. (1998) Vorticity dynamics in a breaking internal gravity wave: 2. Vortex interactions and transition to turbulence. Journal of Fluid Mechanics, 367, 47- 65. https://doi.org/10.1017/S0022112098001645

Fritts, D.C., Vadas, S.L., \& Yamada, Y. (2002). An estimate of strong local body forcing and gravity wave radiation based on $\mathrm{OH}$ airglow and meteor radar observations. Geophysical Research Letters, 29 (10), 71-1 - 71-4. https://doi.org/10.1029/2001GL013753

Fritts, D.C., Wang, L., Werne, J., Lund, T., \& Wan, K. (2009a). Gravity wave instability dynamics at high Reynolds numbers: 1. Wavefield evolution at large amplitudes and high frequencies. Journal of Atmospheric Science 66 (5), 1,126-1,148.

http://dx.doi.org/10.1175/2008JAS2726.1

Fritts, D.C., Wang, L., Werne, J., Lund, T., \& Wan, K. (2009b) Gravity wave instability dynamics at high Reynolds numbers: 2. Turbulence evolution, structure, and anisotropy. Journal of Atmospheric Science 66 (5), 1,149-1,171. https://doi.org/10.1175/2008JAS2726.1

Fritts, D. C., Wang, L., \& Werne, J. (2013). Gravity wave-fine structure interactions, part 1: Influences of fine structure form and orientation on flow evolution and instability. Journal of the Atmospheric Sciences, 70(12), 3710-3734. https://doi.org/10.1175/JAS-D-13-055.1

Fritts, D.C., Baumgarten, G., Wan, K., Werne, J., \& Lund, T. (2014). Quantifying KelvinHelmholtz instability dynamics observed in noctilucent clouds: 2. Modeling and interpretation of observations. Journal of Geophysical Research: Atmospheres, 119(15), 9,359-9,375.

https://doi.org/10.1002/2014JD021833

Fritts, D.C., Wang, L., Baumgarten, G., Miller, A.D., Geller, M.A., Jones, G., et al. (2017). High-resolution observations and modeling of turbulence sources, structures, and intensities in the upper mesosphere. Journal of Atmospheric and Solar-Terrestrial Physics, 162, 57-78. https://doi.org/10.1016/j.jastp.2016.11.006

Fritts, D. C., Vosper, S. B., Williams, B. P., Bossert, K., Plane, J. M. C., Taylor, M. J., et al. (2018). Large-amplitude mountain waves in the mesosphere accompanying weak crossmountain flow during DEEPWAVE Research Flight RF22. Journal of Geophysical Research: Atmospheres, 123, 9992- 10,022. https://doi.org/10.1029/2017JD028250

Fritts, D.C., Dong, W., Lund, T.S., Wieland, S.A., \& Laughman, B. (2019). Selfacceleration and instability of gravity wave packets: 3 . Three-dimensional packet propagation, secondary gravity waves, momentum transport, and transient mean forcing in tidal winds. 
Journal of Geophysical

Research:

Atmospheres,

(3), https://doi.org/10.1029/2019JD030691

Fritts, D.C., Miller, A.D., Kjellstrand, C.B., Geach, C., Williams, B.P., Kaifler, B., et al. (2019). PMC Turbo: Studying gravity wave and instability dynamics in the summer mesosphere using polar mesospheric cloud imaging and profiling from a stratospheric balloon. Journal of Geophysical Research: Atmospheres, 124, 6,423-6,443. https://doi.org/10.1029/2019JD030298

Fritts, D.C, Wang, L., Taylor, M.J., Pautet, P.-D., Criddle, N.R., Kaifler, B., et al. (2019). Large amplitude mountain waves in the mesosphere observed on 21 June 2014 during DEEPWAVE: 2. Nonlinear dynamics, wave breaking, and instabilities. Journal of Geophysical Research: Atmospheres, 124, 10,006-10,032. https://doi.org/10.1029/2019JD030932

Gadsden, M. \& Shröder, W. (1989). Noctilucent clouds, Berlin, Germany: Springer-Verlag

Garcia, R. R., López-Puertas, M., Funke, B., Marsh, D. R., Kinnison, D. E., Smith, A. K., \& González-Galindo, F. (2014). On the distribution of CO2 and CO in the mesosphere and lower thermosphere. Journal of Geophysical Research: Atmospheres, 119, 5700-5718. https://doi.org/10.1002/2013JD021208

Geller, M. A., Alexander, M. J., Love, P. T., Bacmeister, J., Ern, M., Hertzog, et al. (2013). A comparison between gravity wave momentum fluxes in observations and climate models. Journal of Climate, 26, 6,383- 6,405. https://doi.org/10.1175/JCLI-D-12-00545.1

Hecht, J.H., Walterscheid, R.L., \& Vincent, R. A. (2005) Airglow observations of dynamical (wind shear-induced) instabilities over Adelaide, Australia, associated with atmospheric gravity waves, Journal of Geophysical Research: Atmospheres, 106, 28,189$28,197$.

https://doi.org/10.1029/2001JD000419

Hines, C. O. (1960). Internal atmospheric gravity waves at ionospheric heights, Canadian Journal of Physics, 38, 1441, https://doi.org/10.1139/p60-150

Kaifler, N., Kaifler, B., Ehard, B., Gisinger, S., Dörnbrack, A., Rapp, M., et al. (2017) Observational indications of downward-propagating gravity waves in middle atmosphere lidar data, Journal of Atmospheric and Solar-Terrestrial Physics, Volume 162, 16-27, https://doi.org/10.1016/j.jastp.2017.03.003

Kaifler, N., Kaifler, B., Wilms, H., Rapp, M., Stober, G., \& Jacobi, C. (2018). Mesospheric temperature during the extreme midlatitude noctilucent cloud event on 18/19 July 2016. Journal of Geophysical Research: Atmospheres, 124, 13,775-13,789. https://doi.org/10.1029/2018JD029717

Kaifler, B., Rempel, D., Roßi, P., Büdenbender, C., Kaifler, N., and Baturkin, V. (2020). A technical description of the balloon lidar experiment BOLIDE. Atmospheric Measurement Techniques, https://doi.org/10.5194/amt-2020-150, Under review 
Kim, Y.-J., Eckermann, S.D, \& Chun H.-Y. (2003) An overview of the past, present and future of gravity-wave drag parameterization for numerical climate and weather prediction models. Atmosphere-Ocean, 41(1), 65-98. https://doi.org/10.3137/ao.410105

Kjellstrand, C.B., Jones, G., Geach, C., Williams, B.P., Fritts, D.C, Miller, A., et al. (2020) The PMC Turbo balloon mission to measure gravity waves and turbulence in Polar Mesospheric Clouds: Camera, telemetry, and software performance. Earth and Space Science, Unpublished manuscript, https://doi.org/10.1029/2020EA001238

Lane, T.P \& Sharman, R.D. (2006). Gravity wave breaking, secondary wave generation, and mixing above deep convection in a three-dimensional cloud model. Geophysical Research Letters, 33, L23813. https://doi.org/10.1029/2006GL027988

Lehmacher, G. A., Guo, L., Kudeki, E., \& Chau, J. (2007). High-resolution observations of mesospheric layers with the Jicamarca VHF radar. Advances in Space Research, 40, 734-743. https://doi.org/10.1016/j.asr.2007.05.059

Lelong, M.-P. \& Dunkerton, T.S. (1998). Inertia-gravity wave breaking in three dimensions: 1. Convectively stable waves. Journal of Atmospheric Sciences, 55, 2,473-2,488. https://10.1175/1520-0469(1998)055<2473:IGWBIT>2.0.CO;2

Lindzen, R.S. (1981). Turbulence and stress owing to gravity wave and tidal breakdown. Journal of Geophysical Research: Oceans, 86 (C10), 9,707-9,714.

https://doi.org/10.1029/JC086iC10p09707

Lombard, P.N. \& Riley, J.J. (1996) Instability and breakdown of internal gravity waves: 1. Linear stability analysis. Physics of Fluids, 8(12), 3,271-3,287. https://doi.org/10.1063/1.869117

Lübken, F.-J., Rapp, M., \& Hoffman, P. (2002). Neutral air turbulence and temperatures in the vicinity of polar mesospheric summer echoes. Journal of Geophysical Research: Atmospheres, 107, D15, ACL 9-1-ACL 9-10. https://doi.org/10.1029/2001JD000915

Luce, H., Kantha, L., Yabuki M., \& Hashiguchi, H. (2018). Atmospheric Kelvin-Helmholtz billows captured by the MU radar, lidars and a fish-eye camera. Earth, Planets and Space, 70, 162. https://doi.org/10.1186/s40623-018-0935-0

Mccomas, C. H., \& Bretherton, F. P. (1977). Resonant interaction of oceanic internal waves. Journal of Geophysical Research: Oceans and Atmospheres, 82, 1,397-1,412. https://doi.org/10.1029/JC082i009p01397

Miller, A.D., Fritts, D.C., Chapman, D., Jones, G., Limon, M., Araujo, D, et al. (2015). Stratospheric imaging of noctilucent clouds: A new window on small-scale atmospheric dynamics. Geophysical Research Letters, 42 (14), 6,058-6,065. http://dx.doi.org/10.1002/2015GL064758

Nappo, C. (2002). An introduction to atmospheric gravity waves, San Diego, CA: Academic Press. 
Pfrommer, T., Hickson, P., \& She, C.-Y. (2009). A large-aperture sodium fluorescence lidar with very high resolution for mesopause dynamics and adaptive optics studies. Geophysical Research Letters, 36, L15831. https://doi.org/10.1029/2009GL038802

Rapp, M., Lübken, F.-J, Müllemann, A., Thomas, G.E., \& Jensen, E.J. (2002) Small-scale temperature variations in the vicinity of NLC: Experimental and model results. Journal of Geophysical Research: Atmospheres, 107, D19, AAC 11-1 - AAC 11-20. https://doi.org/10.1029/2001JD001241

Satomura, T. \& Sato, K. (1999). Secondary generation of gravity waves associated with the breaking of mountain waves. Journal of the Atmospheric Sciences. 56, 3847-3858. https://doi.org/10.1175/1520-0469(1999)056<3847:SGOGWA>2.0.CO;2

Savigny, C. \& Hoffman, C. (2020). Issues related to the retrieval of stratospheric-aerosol particle size information basedon optical measurements. Atmospheric Measurement Techniques, 13, 4, https://doi.org/10.5194/amt-13-1909-2020

Sonmor, L.J. \& Klaasen, G.P. (1997). Toward a unified theory of gravity wave stability. Journal of the Atmospheric Sciences. 54(22), 2,655-2,680.

https://doi-org.ezp1.lib.umn.edu/10.1175/1520-0469

Strelnikov, B., Rapp, M., Strelnikova, I., Engler, N., \& Latteck, R. (2009). Small-scale structures in neutrals and charged aerosol particles as observed during the ECOMA/MASS rocket campaign. Annales Geophysicae. 27, 1,449-1,456. https://doi.org/10.5194/angeo-271449-2009

Taylor, M.J., Pautet, P.-D., Fritts, D.C., Kaifler, B., Smith, S.M., Zhao, Y., et al. (2019). Large-amplitude mountain waves in the mesosphere observed on 21 June 2014 during DEEPWAVE: 1. Wave development, scales, momentum fluxes, and environmental sensitivity. Journal of Geophysical Research: Atmospheres, 124 (19), 10,364-10,384. https://doi.org/10.1029/2019JD030932

Trackpy v04.1 (2019). https://doi.org/10.5281/zenodo.3492186

Ugolnikov, O.S, Galkin, A.A., Pilgaev, S.V., \& Roldugin, A.V. (2017). Noctilucent cloud particle size determination based on multi-wavelength all-sky analysis. Planetary and Space Science, 146 (15), 10-19. https://doi.org/10.1016/j.pss.2017.08.006

Vadas, S.L., Zhao, J., Chu, X., \& Becker, E. (2018). The excitation of secondary gravity waves from local body forces: theory and observation. Journal of Geophysical Research: Atmospheres, 123 (17), 9,296-9,325. https://doi.org/10.1029/2017JD027970

Vincent, R. (2015) The dynamics of the mesosphere and lower thermosphere: A brief review. Progress in Earth and Planetary Science, 2, 4, https://doi.org/10.1186/s40645-0150035-8

Witt, G. (1962). Height, structure and displacements of noctilucent clouds. Tellus. 14, 118. https://doi.org/10.3402/tellusa.v14i1.9524 
Zasetsky, A.Y., Petelina, S.V., Remorev, R., Boone, C.D., Bernath, P.F., \& Llewellyn, E.J. (2009). Ice particle growth in the polar summer mesosphere: Formation time and equilibrium size. Geophysical Research Letters, 36 (15), L15803. https://doi.org/10.1029/2009GL038727

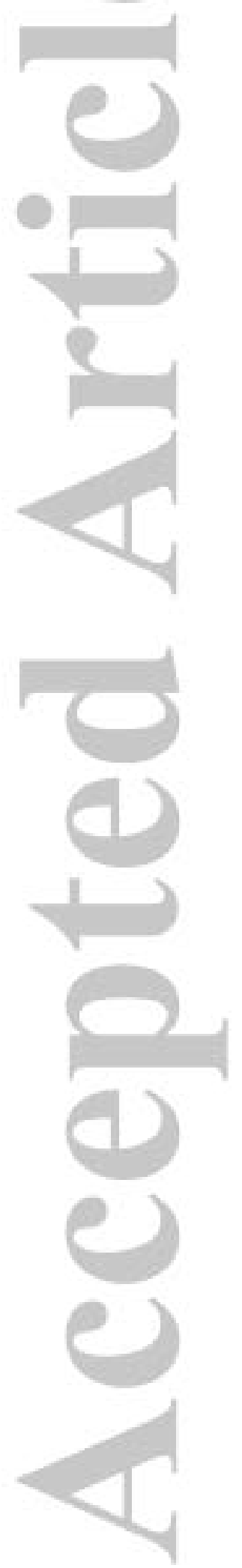




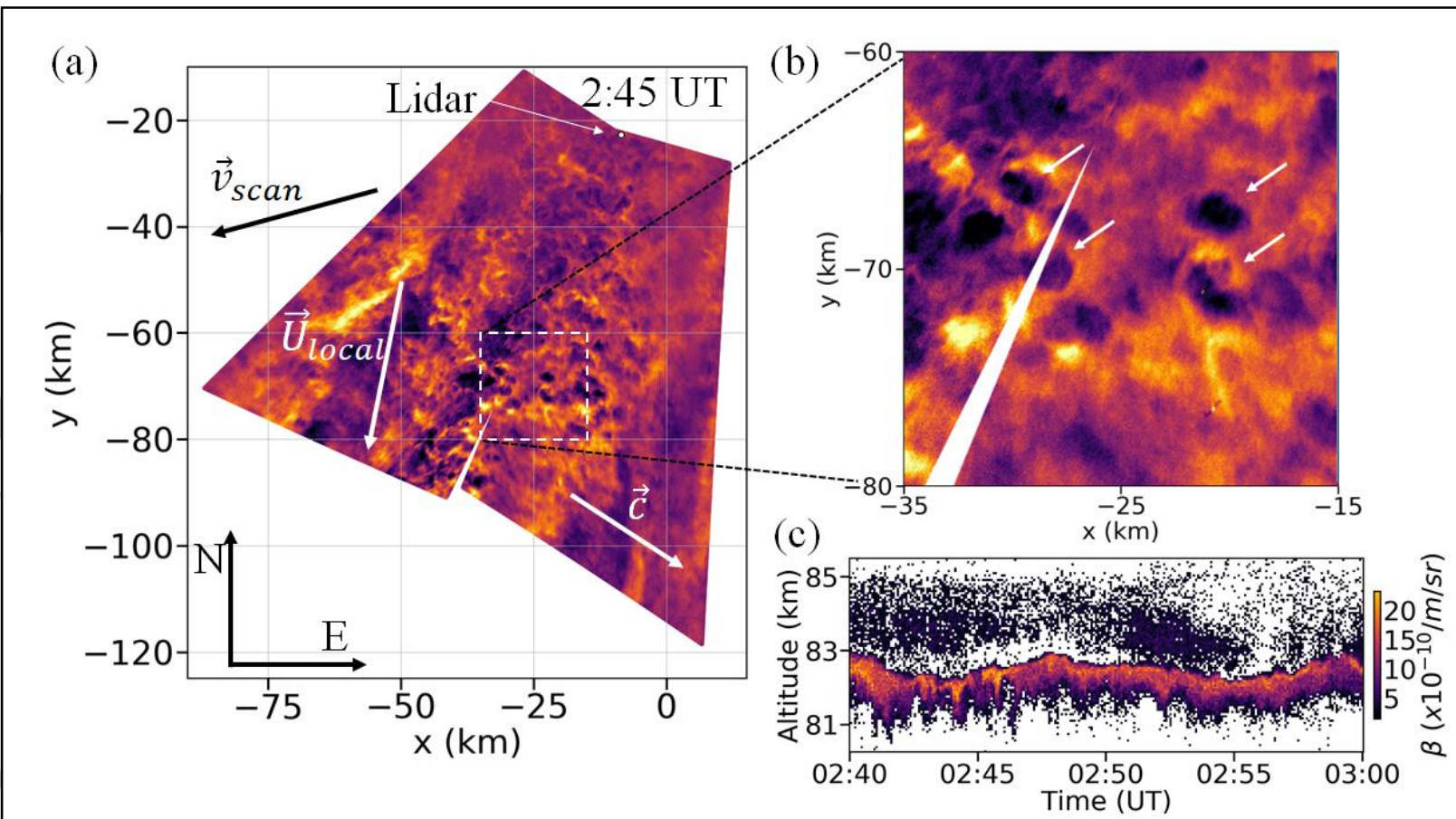

Figure 1. (a) PMC Turbo image data at 2:45 UT on 10 July 2018. The vectors $\vec{U}_{\text {local }}$ and $\vec{c}$ are the local wind speed as inferred from the motion of features between successive images (Section 3.3.2) and the phase speed of the GW as inferred from the advection of the bright phase lines (Section 3.1), respectively. A white dot on the northern edge of the FOV marks the location of the lidar beam, and $\vec{v}_{\text {scan }}$ gives the direction in which the lidar scans (a combination of gondola translation and rotation). Images are flatfielded and displayed in false color, proportional to brightness in uncalibrated units. (b) Zoom of a portion of the FOV showing multiple vortex ring structures at a range of scales. (c) PMC backscatter coefficient $\beta$ as measured by the Rayleigh lidar during the event, showing an oscillation in altitude with $\sim 10$ min period, along with sharp downward features seen at early times.

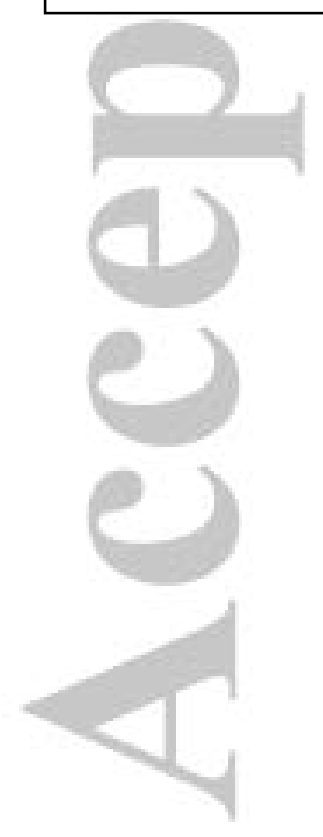



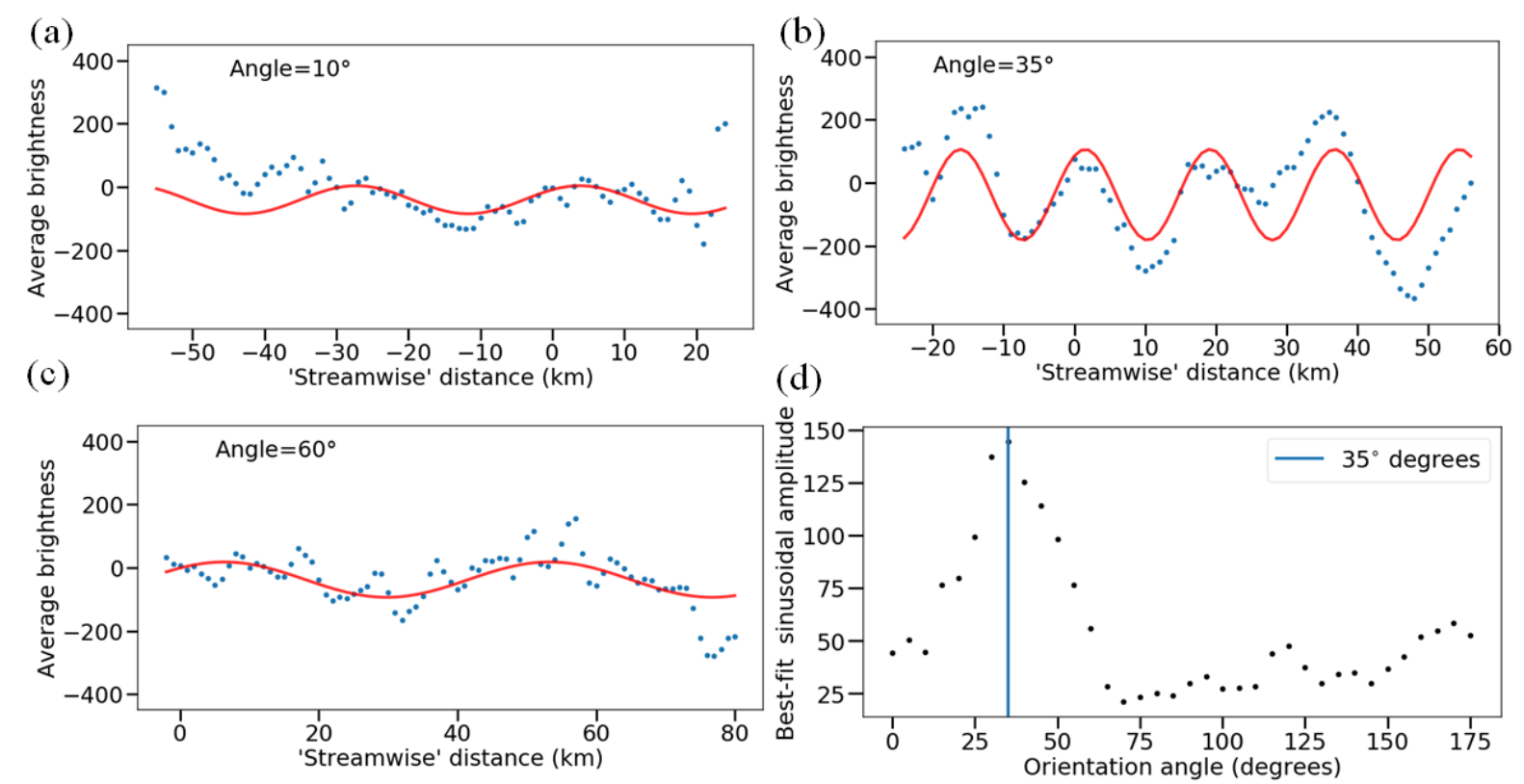

Figure 2. (a-c) Average brightness (blue dots) and sinusoidal fits (red) at orientations of $10^{\circ}$, $35^{\circ}$ and $60^{\circ}$, respectively, plotted as a function of distance perpendicular to the averaging direction. (d) Amplitude of the sinusoidal fit to the binned averaging as a function of orientation angle of the strips. The sharp peak corresponds to the angle at which the averaging is along a common phase of the identified GW. The data used in this analysis were the image data from 2:45 UT, shown in Figure 1. 

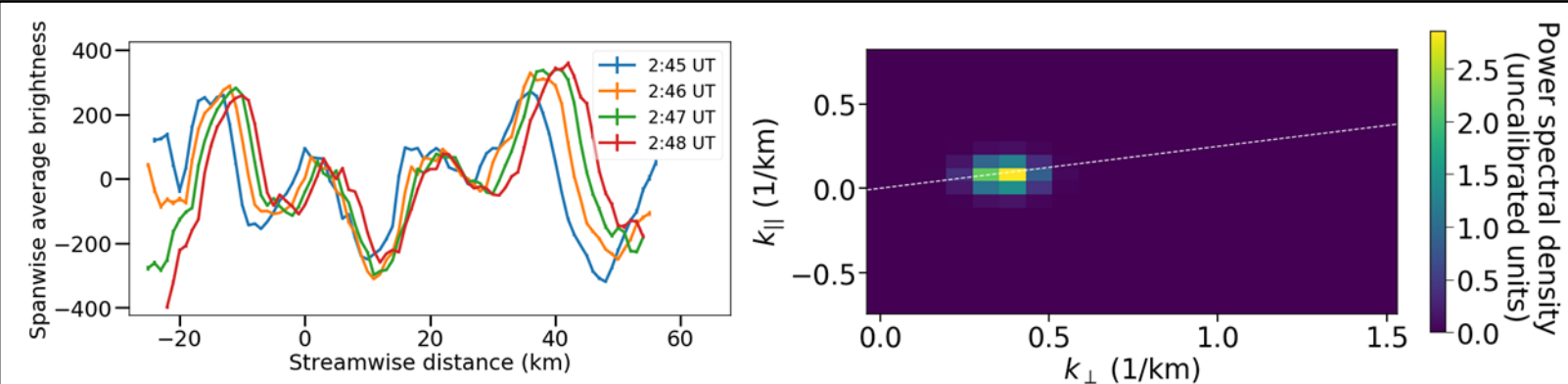

Figure 3. (Left) Series of spanwise averages of image data plotted as a function of distance in the streamwise direction, taken at 1 min intervals and showing the phase progression of the GW. (Right) 2-D power spectrum of the image data at 2:45 UT, showing a peak corresponding to the GW signal found in the spatial domain. Note that the coordinate system is rotated in order to make the image data fit more compactly in a rectangular domain such that the $y$-axis $\left(k_{||}\right)$ corresponds to the anti-sun direction. The dashed white line corresponds to the streamwise direction. 


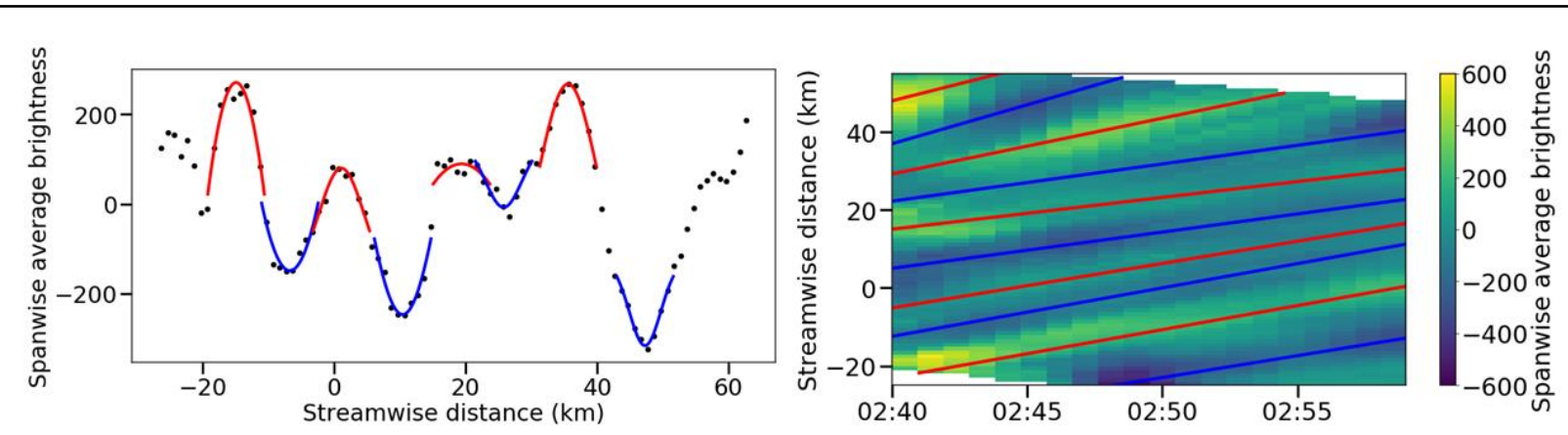

Figure 4. (Left) Example of the spanwise average of the image brightness as a function of distance in the streamwise direction (dots) and the best-fit Gaussian profiles (solid red and blue) for the peaks and troughs. (Right) Color plot of the spanwise average of the image brightness, as a function of time (x-axis) and distance in the streamwise direction (y-axis). Red (blue) fits correspond to peaks (troughs) in the brightness data. 

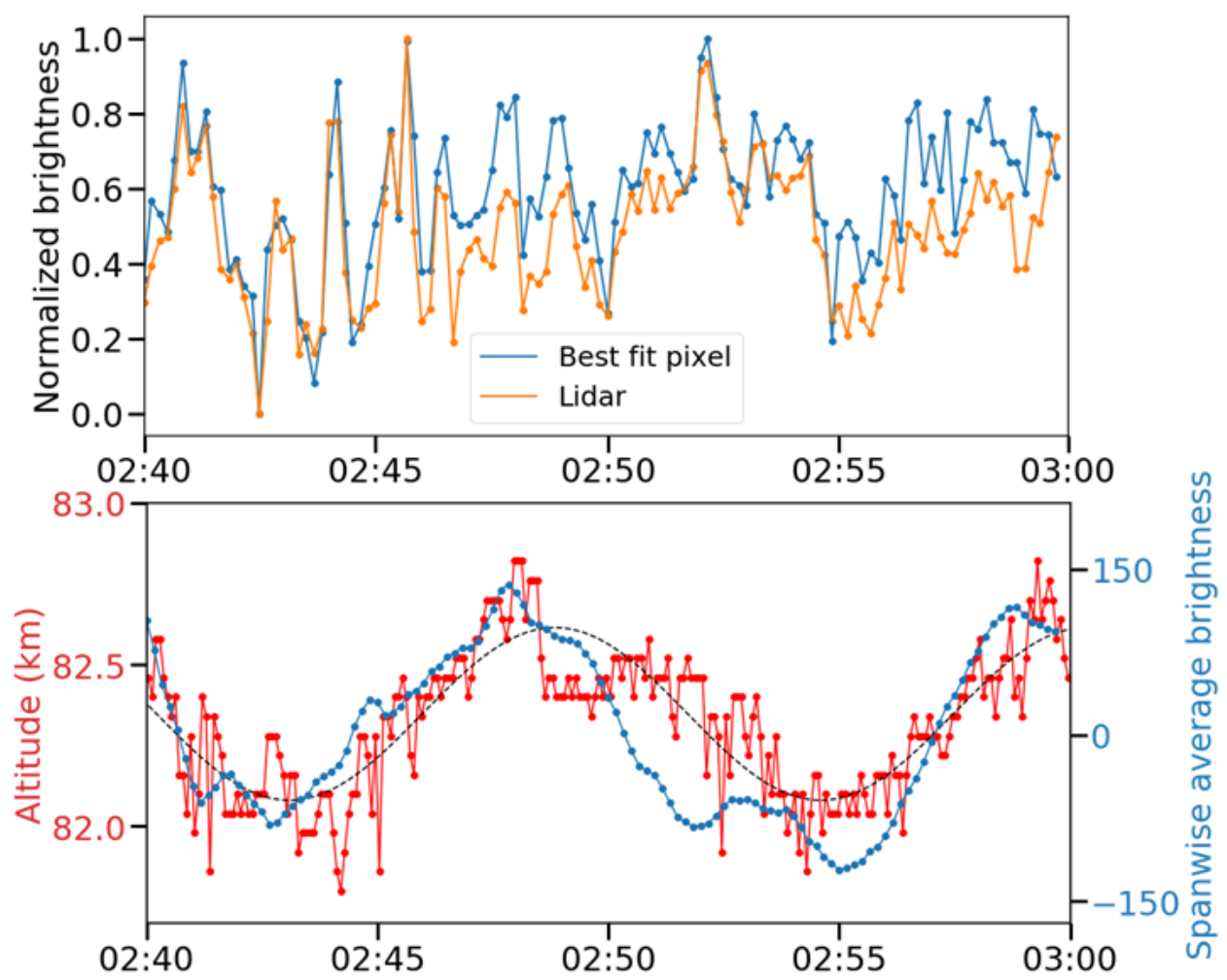

Figure 5. (Top) Integrated PMC brightness as measured by the lidar (orange) and the cameras (blue) at the determined point of overlap between these instruments. Both datasets have been normalized to lie between 0 and 1. (Bottom) PMC layer altitude (red, left ordinate) and best-fit sinusoid (black), and spanwise-averaged brightness at the streamwise location of the lidar beam (blue, right ordinate) as a function of time. 


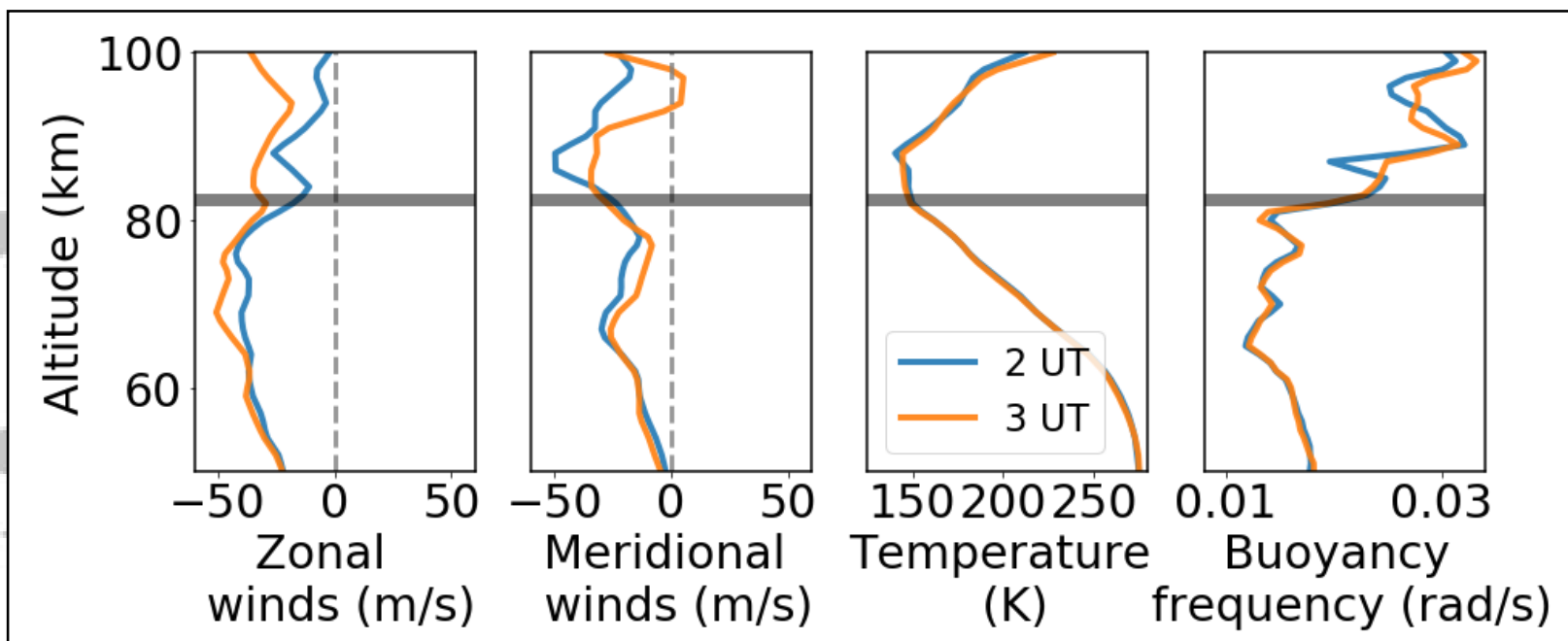

Figure 6. NAVGEM profiles of zonal winds, meridional winds, temperature, and buoyancy frequency averaged horizontally over a $200 \mathrm{~km}$ radius around the location of the gondola during the GW breaking event, at 2 UT (red) and at 3 UT (blue). The gray horizontal bar indicates the approximate altitude of the PMC layer. 

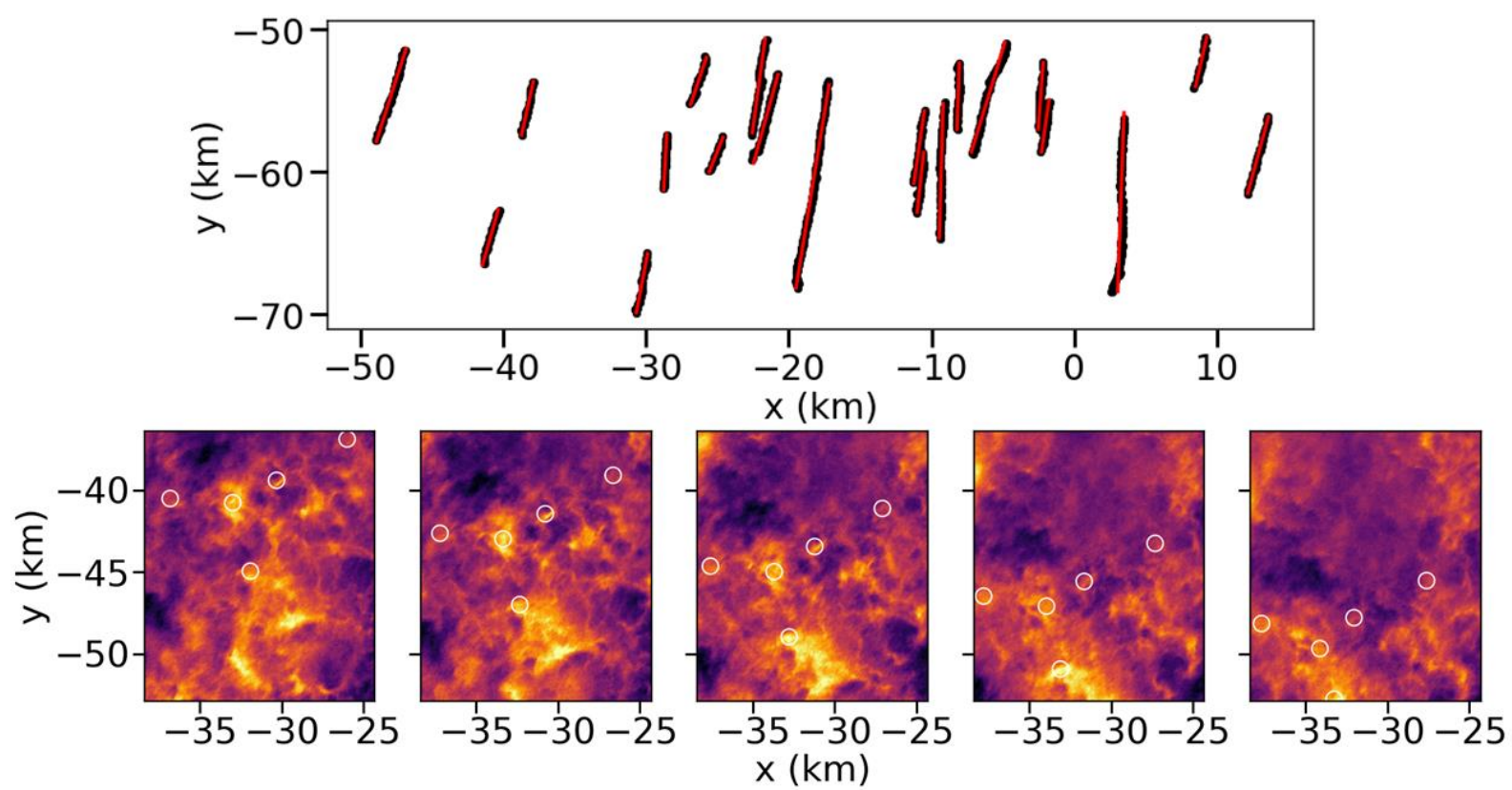

Figure 7. (Top) Scatter plot of a subset of Trackpy trajectories (black dots) and corresponding linear fits (red lines). (Bottom) Zoom of a section of the field of view, starting from 2:45 UT (left) and proceeding in $40 \mathrm{~s}$ intervals, showing the advection of the instability structures. The white circles indicate the locations and approximate size of the features found by Trackpy over this interval. Only features which appear in every frame are shown. 

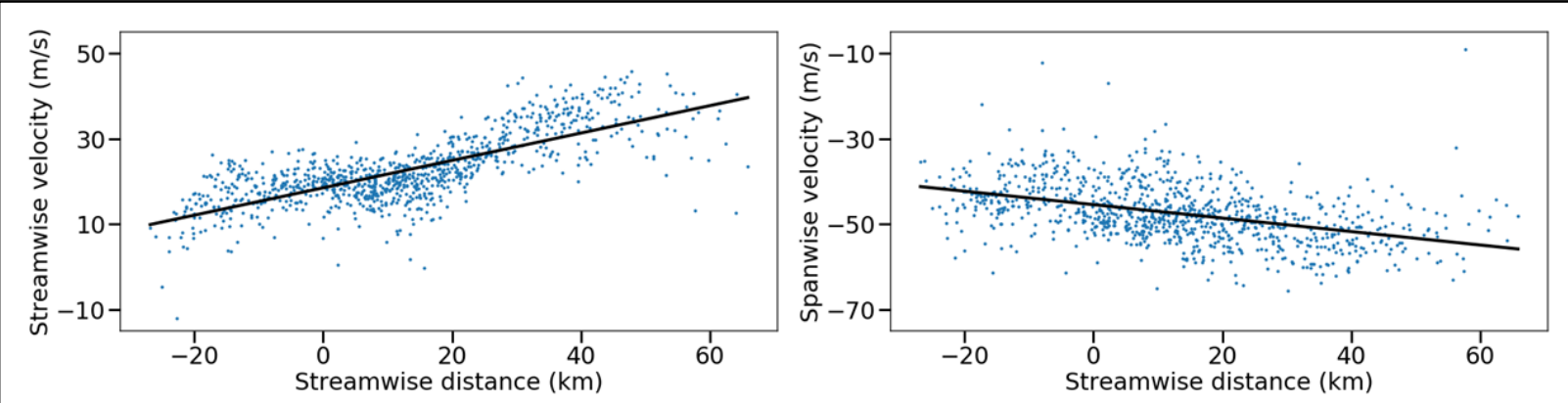

Figure 8. Scatter plots of the streamwise (left) and spanwise (right) trajectory velocities between 2:45 and 2:47 UT as a function of average position in the streamwise direction (blue dots) with linear trends across the field of view (black lines). 

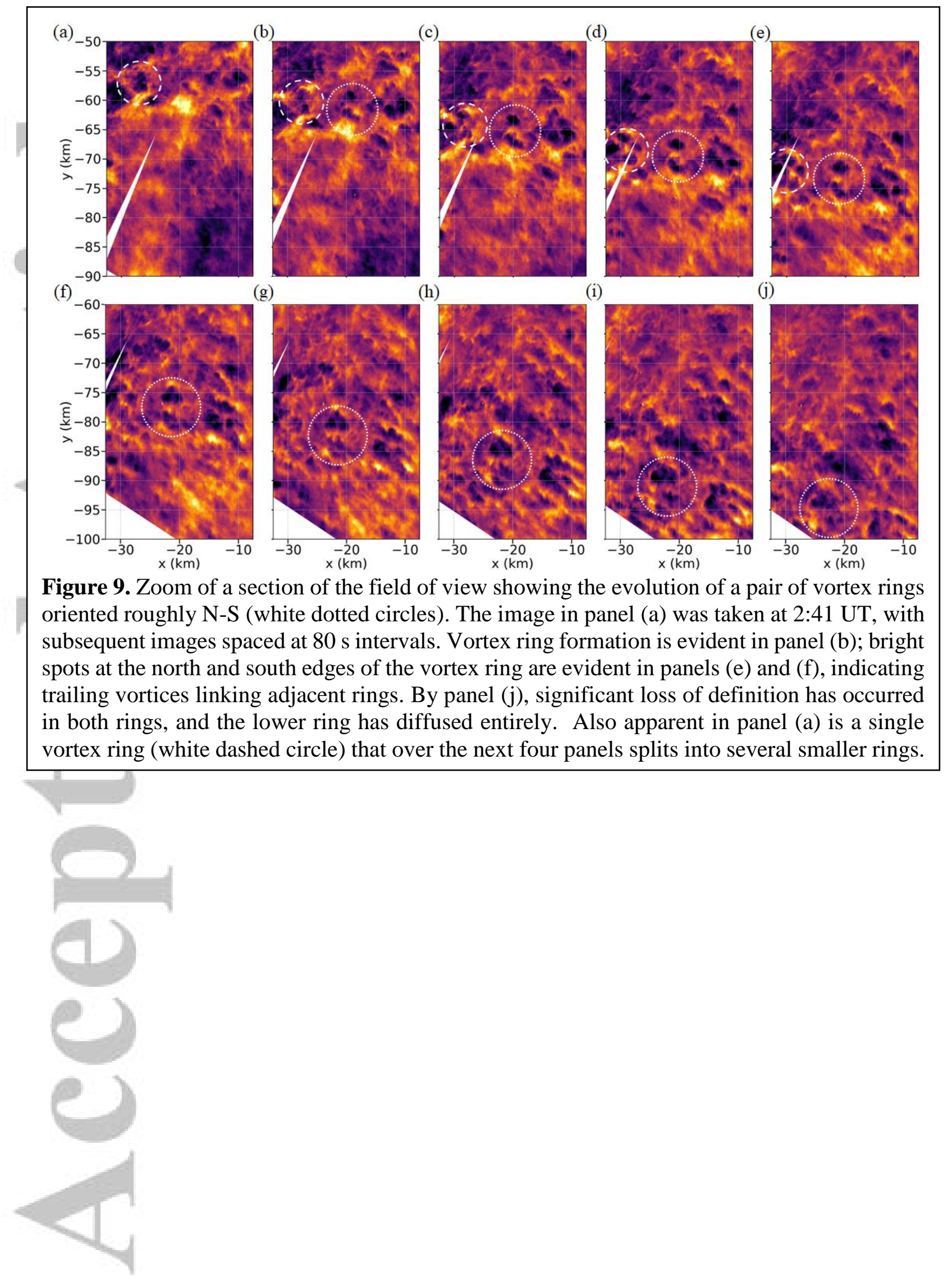


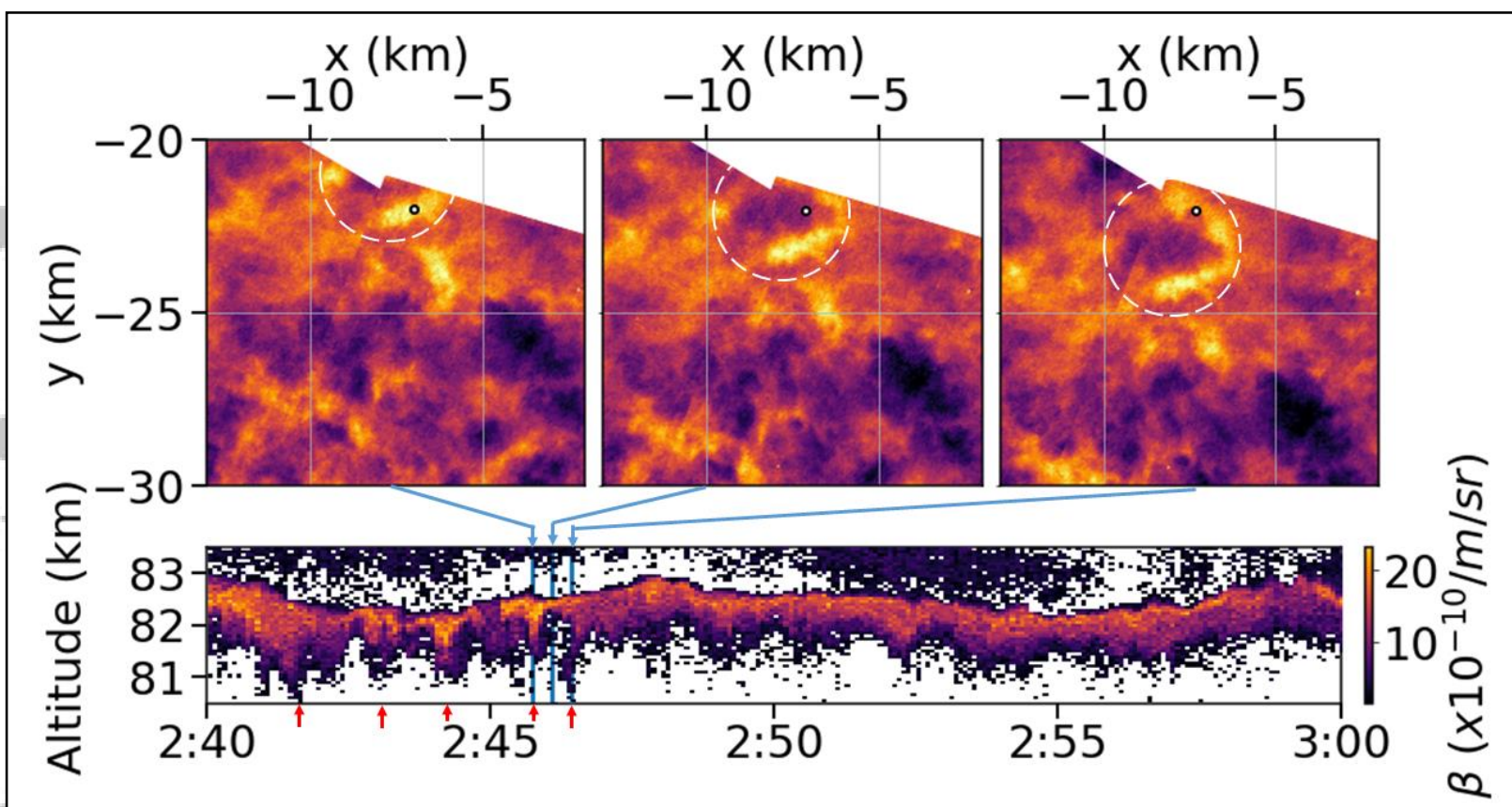

Figure 10 (Top, left to right) Zoomed sections of the camera field of view, starting at 2:45:40 UT (left) and proceeding in $40 \mathrm{~s}$ intervals to the right, with the location of the lidar beam within the field of view (white dot). The images track the passage of a vortex ring through the lidar beam (over-sized white circles). (Bottom) The lidar profile of the GW breaking event, with 


\begin{tabular}{||l|c|l|l||}
\hline GW or Background Parameter & Symbol & Value & Source \\
\hline Mean wind & $\vec{U}_{0}$ & $\left(\begin{array}{l}-28 \pm 9 \\
-29 \pm 4\end{array}\right) \mathrm{m} \mathrm{s}^{-1}$ & NAVGEM \\
\hline Buoyancy frequency & $N$ & $0.02 \pm 0.004 \mathrm{rad} \mathrm{s}^{-1}$ & NAVGEM \\
\hline Buoyancy period & $T_{b}$ & $310 \pm 60 \mathrm{~s}$ & NAVGEM \\
\hline Scale height & $H$ & $4.4 \pm 0.1 \mathrm{~km}$ & NAVGEM \\
\hline Propagation direction & $\hat{k}$ & $\left(\begin{array}{c}0.82 \pm 0.03 \\
-0.57 \pm 0.02\end{array}\right)$ & PMC Turbo \\
\hline Horizontal wavelength & $\lambda_{h}$ & $18 \pm 0.5 \mathrm{~km}^{18}$ & PMC Turbo \\
\hline Vertical wavelength & $\lambda_{z}$ & $9 \pm 4 \mathrm{~km}$ & Linear theory \\
\hline Apparent phase speed & $c$ & $21 \pm 6 \mathrm{~m} \mathrm{~s}^{-1}$ & PMC Turbo \\
\hline $\begin{array}{l}\text { Intrinsic phase speed } \\
\text { Period }\end{array}$ & $c_{i}$ & $27 \pm 8 \mathrm{~m} \mathrm{~s}^{-1}$ & Linear theory \\
\hline Intrinsic frequency & $\omega_{i}$ & $0.009 \pm 0.003 \mathrm{rad} \mathrm{s}^{-1}$ & Linear theory \\
\hline $\begin{array}{l}\text { Table 1. Inferred estimates of relevant GW and background parameters and their source. } \\
\text { Values derived from linear theory are calculated from NAVGEM estimates and PMC Turbo } \\
\text { observations. }\end{array}$ & \\
\hline
\end{tabular}

